\title{
TECNICISMOS JURIDICOS EN UN CÓDIGO DEL FUERO JUZGO
}

\author{
Veronica Orazi \\ (Università degli Studî di Firenze)
}

\begin{abstract}
RESUMEN
This article contains a list of juridical technicalities proceding from a half XIIIth century Fuero Juzgo manuscript in old leonese.

Many therms survive actually, others were substituted by new voices. Their abundance and exact use seem to us the main features to underline, as well as their diffusion and linguistic reality in the XIIIth century.

Such kind of linguistic studies in hispano-romance -about lexical technicalitiesare in our opinion not enough diffused as the interest of the subject would require.
\end{abstract}

Señalamos en este artículo algunos tecnicismos jurídicos de especial interés procedentes de un código del Fuero Juzgo de la segunda mitad del siglo xill, según una versión en leonés antiguo. Son términos documentados que han sobrevivido hasta nuestros días y cuyo empleo es corriente en la actualidad. Otros han desaparecido y su pérdida se ha compensado con voces nuevas. Lo más interesante nos parece, además de la misma atestación de dichos tecnicismos, su empleo sin vacilaciones e incertidumbres y su difusión en el siglo xıI.

El estudio de los tecnicismos jurídicos en hispano-romance merecería sin duda mayor atención de la que se le dedica.

Esta contribución, cuya mayor pretensión sería despertar el interés de otros estudiosos, aportará indudablemente algún aclarecimiento en un dominio bastante desatendido de los estudios filológicos hispánicos. 
accusador < ACCUSATORE, sust. masc. sing., «acusador».

acordadamientre, derivado de CORCORDIS, adv. de modo, "de acuerdo, en conformidad».

acreedor, derivado de CREDERE, sust. masc. sing., «acreedor».

$a c t a<$ ACTA, sust. fem. sing., «acta, documento notarial».

acusacion < ACUSATIONE, sust. fem. sing., «acusación».

acusador, derivado de ACCUSARE, sust. masc. sing., «acusador».

acusamiento, derivado de ACCUSARE, sust. masc. sing., «acusación».

acusar< ACCUSARE, v. trans., «acusar». adepartir, derivado de PARTIRE, v. trans., «partir, dividir».

adesfazer, derivado de FACERE, v. trans., «invalidar, anular, casar».

adulterador, derivado de ADULTERARE, sust. masc. sing., «adúltero».

aduzir < ADDUCERE, v. trans., «aducir, alegar, conducir, llevar».

adversario < ADVERSARIU, sust. masc. sing., «contraparte».

aemendar < EMENDARE, v. trans., «enmendar».

aentregar $<$ INTEGRARE, v. trans., «entregar».

[aescomungar $]<*$ EXCOMUNICARE, $v$. trans., «escomungar».

afirmar <: AFFIRMARE, v. trans., «convalidar, confirmar».

aiuntancia < *ADJUNCTANTIA, sust. fem. sing., «unión».

[alargar] < * ADLARGARE, v. trans., «prolongar, detardar».

allegar < ADLEGARE, v. trans., documentado con el valor de «alegar, añadir, juntar»y «llegar» (<*ADPLICARE).

alongar < *ADLONGARE, v. trans., «prolongar, detardar».

[alugar] <*ADLOCARE, v, trans., «alquilar».

[aministrar] < ADMINISTRARE, v. trans., «administrar».

amonestar $<$ ADMONERE (indirectamente), v. trans., «amonestar».

[anadir] (anadimos) < * INADDERE, v. trans., «alegar, añadir».

anparar < *ANTEPARARE, v. trans., «amparar, proteger».

anteviso < ANTE UISU, adj. masc. sing., «prudente, apercibido».

[apareyar] < *ADPARECULARE, $v$. trans., «preparar».

apartir, derivado de PARTIRE, v. trans., «partir, dividir».

apedrear, derivado de PETRA, v. trans., «apedrear, lapidar».

apellar < APPELLARE, v. intr., «interponer apelación».

apesquerir < PERQUIRERE, v. trans., «indagar, comprobar, averiguar».

apocar, derivado de PAUCU, v. trans., «reducir, disminuir».

[aponer] < ADPONERE, v. trans., «atribuir, atribuirse».

apremiar, relacionado con el latín PREMERE, v. trans., «oprimir, constreñir».

aprovechar, derivado de PROFECTU, v. intr., «aprovechar, disfrutar».

aretraer, derivado de TRAHERE, v. trans., «condonar».

arras < ARRAE, sust. fem. pl., «donación, dote».

[arrendar], derivado de RENDITA, v. trans., «alquilar».

asmar <*ADESTIMARE, v. trans., «avalutar, estimar», efr. osmar.

atemplamiento, derivado de TEMPERARE, sust. masc. sing., «acción y resultado de mitigar, moderar».

atenplançia $<$ TEMPERANTIA, sust. fem. sing., «moderación, templanza».

autentico < AUTENTICU, adj. masc. sing., «auténtico».

avenencia, derivado de *ADVENIRE, sust. fem. sing., «acuerdo, compromiso».

[aviesso], < ADUERSU, adj. masc. sing., «adverso, contrario, parte contraria».

[azotar], del árabo sáut, v. trans., «azotar, fustigar».

[barragan], probablemente de origen germánico, sust. masc. sing., «joven, querido, concubino».

beneficio < BENEFICIU, sust. masc. sing., «beneficio, ventaja».

blasmar, derivado de BLASPHEMU, $v$. trans. e intr., documentado en el sentido de «difamar, infamar» y de «blasfemar». 
bona, buena < BONA, sust. fem. sing., documentado con valor colectivo originario de «pertenencias, bienes».

cabildo < CAPITULU, sust. masc, sing., «capítulo eclesiástico».

caldaria (ley) < CALDARIA, adj. fem. sing., la ley caldaria o prueba judiciaria consistía en una prueva a la que se sometía el presumido reo, en el caso de que éste resultara indemne estaba provada su inocencia.

calomia < CALUMNIA, sust. fem. sing., «calumnia».

canbiar < CAMBIARE, v. trans., «cambiar, baratar».

carta < CHARTA, sust. masc. sing., «documento, carta, mensaje».

casamiento, derivado de CASA, sust. masc. sing., «casamiento».

castigar < CASTIGARE, v, trans., «castigar».

[cativar $]<$ CAPTIUARE, v. trans., «capturar».

[caudelador $]<$ CAPITELLATORE, sust. masc. sing., «caudillo».

cobrar < RECUPERARE, v. trans., «cobrar, recobrar»; cfr. combrar

[comandamiento], derivado de MANDARE, sust. masc. sing., "preceptos, mandamientos».

comarca, de origen germánico, sust. fem. sing., «zona, región colindante».

[comarcar], de origen germánico, v. intr., «confinar».

combrar, conbrar < RECUPERARE, v. trans., «cobrar, recobrar».

comienda < COMMENDA, sust. fem. sing., en la expresión en comienda significa «bajo tutela».

composicion < COMPOSITIONE, sust. fem. sing., «acuerdo».

comprador, derivado de COMPERARE, v. trans., «comprador».

comtencion $<$ CONTENTIONE, sust. fem. sing., «contienda, disputa, altercación».

conceyo $<$ CONCILIU, sust. masc. sing., «concilio, consejo».

condenar < CONDEMNARE, v. trans., «condenar».

confirmar $<$ CONFIRMARE, v. trans., «confirmar, convalidar». [conrompedor], derivado de CORRUM PERE, sust. masc. sing., «corruptor». consentemiento $<*$ CONSENTIMENTU, sust. masc. sing., «consentimiento, asentimientos.

consentir, derivado de CONSENSU, $v$. intr., «consentir».

constitucion < CONSTITUTIONE, sust. fem. sing., «constitución».

contienda, derivado de CONTENDERE, sust. fem. sing., «contienda, pleito».

contradezir, derivado de DICERE, v. trans., «contredecir, desmentir».

contrariar, derivado de CONTRA, v. trans., «contrariar, adversar».

[contrastar $]<$ CONTRASTARE, v. trasn., «contrastar».

convenencia, derivado de CONUENIRE, sust. fem. sing., «acuerdo, compromiso».

corrompemiento, derivado de CORRUMPERE, sust. masc. sing., «acción y resultado de corromper».

corromper < CORRUMPERE, v. trans., «corromper».

[costa], derivado de CONSTARE, sust. fem. sing., «Cuesto, precio, gasto».

costrenir, derivado de STRINGERE, v. trans., «constreñir».

criancia, derivado de CRESCERE, sust. fem. sing., «prole».

culpa < CULPA, sust. fem. sing., «culpa».

culpar, derivado de CULPA, v. trans., «enculpar, acusar».

cuncta $<{ }^{*}$ COMPUTA, sust. fem. sing., «cálculo, cuento».

[dannar], derivado de DAMNU, v. trans., «perjudicar, dañar».

debda, deoda, deveda < DEBITA, sust. fem. sing., «deuda».

debdor, deodor, deudor, devedor $<\mathrm{DEBI}-$ TORE, sust. masc. sing., «deudor».

decaemiento, derivado de CADERE, sust. masc. sing., «decaimiento».

decibimiento, derivado de DECIPERE, sust. masc. sing., «engaño».

declarar $<$ DECLARARE, v, trans., «declarar».

decreto, degredo < DECRETU, sust. masc. sing., «degredo».

defendedor, derivado de DEFENDERE, 
sust. masc. sing., «defensor, abogado».

defendemiento, derivado de DEFENDERE, sust. masc. sing., documentado con el valor de «defensa» y de «prohibición».

defender $<$ DEFENDERE, v. trans., documentado con el valor de «defender» $y$ de «prohibir».

defurtadamientre, derivado de FURTU, adv. de modo, «ocultamente, a escondidass.

delibrar, delivrar < DELIBERARE, $v$. trans., «juzgar, establecer».

demanda, derivado de DEMANDARE, sust. fem. sing., documentado con el valor de «instancia» y de «reivindicación».

[demandador], derivado de DEMANDARE, sust. masc. sing., «acreedor».

demandar < DEMANDARE, v. trans., «reciamar, reivindicar».

demorancia <*DEMORANTIA, sust. fem. sing., «dilación, retraso».

demostrar, derivado de MONSTRARE, v. trans., «demostrar, probar».

departemiento < $<$ DEPARTIMENTU, sust. masc. sing., «separación», también «partición, división, subdivisión».

departir, derivado de PARTIRE, v. trans., «separar, disolver, dividir».

desanparar, derivado de *ANTEPARARE, v. trans., «abandonar, desamparar».

[desavinimiento], derivado de ADUENIRE, sust. masc. sing., «desacuerdo».

descabezar, derivado de *CAPITIA, v. trans., «decapitar».

descomungar < DEXCOMUNICARE, $v$. trans., «excomulgar».

descomunion, derivado de COMMUNIONE, sust. fem. sing., «excomunión».

deseredar, derivado de HEREDITARE, $v$. intr., «deseredar».

[desfamar $\mid<$ *DEXFAMARE, v. trans., «infamar».

[desfollar] < *DEXFOLIARE, v. trans., «desollar, despellejar».

[desgastar], derivado de *WASTARE, v. trans., «devastar, dañar».

[deslapredar], de origen incierto, probablemente de lapo (latigazo, garrotazo), v. trans., «desfigurar, perjudicar».

deslaydar, del francés arcáico laid, v. trans., «herir, desfigurar, perjudicar».

desondrar $<*$ DEXHONORARE, v. trans., «deshonrar».

[despoyar $]<$ DEXPOLIARE, v. trans., «privar, desposeer».

despreciamiento, derivado de PRETIU, sust. masc. sing., «desprecio, acción y resultado de despreciar».

despreciar, derivado de PRETIU, v. trans., «despreciar».

desprecio, derivado de PRETIU, sust. masc. sing., «desprecio, acción y" resultado de despreciar».

desputacion, derivado de DISPUTARE, sust. fem. sing., «disputa, controversia»; cfr. disputacion.

desputar < DISPUTARE, v. intr., «disputar, discutir».

desquitar, derivado de QUIETU, v. trans., «condonar».

[desterrar], derivado de TERRA, v. trans., «desterrar, exiliar».

destayar < *DEXTALEARE, v. trans., «prevenir, evitar».

destorvar < DISTURBARE, $v$. trans., «molestar, disturbnar».

destorvo, derivado de DISTURBARE, sust. masc. sing., «molestia».

detardar, derivado de TARDARE, v. intr., «aplazar, detardar, diferir».

[detener], derivado de TENERE, $\vee$. trans., «detener, retener».

determinar $<$ DETERMINARE, v. trans., «cesar un proceso, ocuparse de un pleito».

[dezmar $]<{ }^{*}$ DECIMARE, v. trans., «exigir, cobrar la décima».

diezmo < DECIMU, sust. masc. sing., «décima, impuesto correspondiente a un décimo de los bienes en cuestión».

discordia, derivado de DISCORDARE, sust. fem. sing., «desacuerdo».

disputacion, derivado de DISPUTARE, sust. fem. sing., «disputa, controversia»; cfr. desputacion.

doblar, derivado de DUPLU, v. trans., «doblar».

doblo, dublo, [duplo $]<$ DUPLU, sust. masc. sing., «doble».

donacion < DONATIONE, sust. fem, sing., «donación». 
donador < DONATORE, sust. masc, sing., «onador».

[donar $]<$ DONARE, v. trans, «donar».

dubdoso < "DUBITOSU, adj. masc. sing., «dudoso, incierto».

duenno < DOMINU, sust. masc. sing., «dueño, propietario».

echar, [enechar] < JECTARE, v. Irans., «echar, despedir».

eleccion < ELECTIONE, sust. fem. sing., «elección».

embargo, derivado de "IMBARRICARE, sust. masc. sing., «impedimento».

emendar < EMENDARE, v. trans., «enmendar, corregir, mejorar».

emienda, derivado de EMENDARE, sust. fem. sing., «enmienda».

empeezer < IMPEDIRE, v. intr., «impedir, causar dificultades».

empiezo, derivado de IMPEDIRE, sust. masc. sing., «impedimiento, inconveniente».

[emprestar], derivado de PRAESTARE, v. trans., «prestar».

[enaienamiento], derivado de ALIENARE, sust. masc. sing., «acción y resultado de enajenar» (con referencia a bienes y pertenencias).

enayenar $<$ ALIENARE, v. trans., «enajenar».

[encarçerar], derivado de CARCERE, $v$. trans., «aprisionar, encarcelar, arrestar».

encerrador, derivado de SERRARE, sust. masc. sing., «secuestrador».

encerramiento, derivado de SERRARE, sust. masc. sing., «embargo, secuestro».

encesto < INCESTU, sust. masc. sing., «incesto».

[encobridor], derivado de COOPERIRE, sust. masc. sing., «ocultador».

encobrir, derivado de COOPERIRE, $v$. trans., «ocultar, encobrir».

|encomendamiento $\mid$, derivado de COMMENDARE, sust. masc. sing., «encargo».

[encomendar] < INCOMMENDARE, $v$. trans., "encargar, confiar».

[enculpar], derivado de CUPLA, v. trans., «acusar, enculpar».

engannar < "INGANNARE, v. trans., «defraudar, estafar, engañar». enpennar, derivado de PIGNU, v. trans., «empeñar».

[enriçar], [enrizar], de origen incierto, $v$. trans., literalmente «encrespar, rizar», documentado con el valor de «instigar, azuzar, molestar»; cfr. [enrizar].

entenciar < *INTENTIARE, v. trans.. «citar, entablar pleito».

entencion < *INTENTIONE, sust. fem. sing., «pleito, causa» documentado también con el valor de «entención».

entregar, derivado de INTEGRU, v. trans., «entregar, dar».

[eredar $]<$ HEREDITARE, v. trans., «heredar».

eredat, derivado de HEREDITARE, sust. fem. sing., «heredad».

[eredero], derivado de HEREDITARE, sust. masc. sing., «heredero»; efr. heredero.

[erege], del occitánico eretge. sust. masc. sing., «hereje»; cfr. [hereche], [herege]. escapar < * EXCAPPARE, v. intr., documentado con el valor de «salvarse»y de «escapar, huir».

escomonion, derivado de COMMUNIONE, sust. fem. sing, «excomunión».

[escomungar], derivado de COMMUNICARE, v. trans, «excomulgar».

escripto < SCRIPTU, sust. masc. sing., «documento».

escriptura, derivado de SCRIBERE, sust. fem. sing., «documento».

escrivan, derivado de SCRIBERE, sust. masc. sing., «copista».

escusacion, derivado de EXCUSARE, sust. fem. sing., «atenuante, excusación, justificación».

[esleer] < EXLEGERE, v. trans., «elegir».

esplanar < ${ }^{*}$ EXPLANARE, v. trans, «aclarar, allanar».

[esposaya $]<$ SPONSALIA, sust. fem. sing., «bodas».

[establecemiento!, estavlescemiento, derivado de $S T A B I L E$, sust. masc. sing., «ley, ordenanza, estatuto»; cfr. estavlescemiento.

establecer, derivado de STABILE, $\vee$, trans., «establecer».

estado < STATU, sust. masc. sing., kestado». 
estunar $<$ EXTUNDERE, v. trans., «constreñir».

façedor, derivado de FACERE, sust. masc. sing., en la expresión façedor de las leys significa «legislador, juez».

[falsar], derivado de FALSU, v. trans., «alsificar».

falsario, derivado de FALSU, sust. masc. sing., «falsario».

fazemiento, derivado de FACERE, sust. masc. sing., «acción de preparar y administrar».

[fazferir $]<{ }^{*}$ FACIE FERIRE, v. trans., «imputar repetidamente, reprochar».

fiado, derivado de FIDARE, sust. masc. sing., «garantía».

fiador < *FIDATORE, sust. masc. sing., «garante, fiador».

firmar < FIRMARE, v. trans., «firmar».

fito < FICTU, sust. masc. sing., «mojón o poste para indicar los confines entre dos haciendas».

fondo < FUNDO, sust. masc. sing., en la expresión en fondo tiene el valor de «al pié de la pagina, en fondo».

forciador < *FORTIATORE, sust. masc. sing., «estuprador».

forciar < FORTIARE, v. trans., «estuprar, violentar».

[fostigar $\mid<*$ FUSTIGARE, v. trans. «azotar».

franqueamiento, derivado del germánico frank, sust. masc. sing., «acción y resultado de franquear».

franquear, del germánico frank, v. trans., «franquear".

franquedumne, del germánico frank más el sufijo latín -UDINE > -UMINE, sust. lem. sing., «condición del franqueado».

franquezia, del germánicofrank, sust. fem. sing., "condición del franqueado».

furtar, derivado de FURTU, v. trans., «hurtar, robar».

furto < FURTU, sust. masc. sing., «robo».

fuydizo, derivado de FUGERE, adj. masc. sing., «fugitivo, huidizo».

galardon, de origen germánico, sust. masc. sing., «galardon».

ganancia, del gótico *ganan, sust. fem. sing., «ganancia, provecho». garda, del germánico wardon, sust. fem. sing., «protección, amparo».

gardar, guardar, del germánico wardon, v. trans., «controlar, amparar, proteger».

[gastador] < ${ }^{*} \mathrm{UAST} A T O R E$, pronunciado *wastator por influjo del germánico *wôst(j)an, sust masc. sing., «devastador, saqueador»》.

gastar < *WASTARE, v. trans., documentado con el valor de «gastar»e di «devastar, saquear».

generacion < GENERATIONE, sust. fem. sing., «generación, descendencia».

governador, derivado de GUBERNARE, sust. masc. sing., «gobernador».

governar < GUBERNARE, v. trans., «gobernar, mandar».

gracia < GRATIA, sust. masc. sing., «gracia».

grandable, derivado de GRANDE, adj. masc. sing., «acrecentable».

guardador, derivado del germánico wardon, sust. mesc. sing., «carcelero».

guarnicimiento, del germánico n’ajan, sust. masc. sing., «amparo, defensa, protección».

guarnimiento, del germánico warjan, sust. masc. sing., <amparo, defensa, protección».

[guarnir], del germánico warjan, v. trans., «equipar, proveer».

guerear, del germánico werra, v. trans., «saquear».

guisa, del germánico wisa. sust. fem. sing. «condición, clase social».

[hereche], |herege $\mid$, del occitánico erctge, sust. masc. sing., «hereje»; cfr. leregcl.

[heredade $\rfloor$, derivado de HEREDITARE, sust. fem. sing., «heredad».

heredamiento, derivado de HEREDITARE, sust. masc. sing., "heredad».

heredar < HEREDITARE, v. trans., «heredar».

heredero, derivado de HEREDITARE, sust. masc. sing., «heredero»; cfr. [erederol.

homicio < HOMICIDIU. sust. masc. sing., «homicidio».

hoste, hueste < HOSTE. sust. fem. sing., «ejercito»; clit mueste.

[husura $]<$ USURA. sust. fon. sing., «usura»; chr. astra. 
infazon < INFANTIONE, sust. masc. sing., «hidalgo de los pertenecientes a cierta clase que tenía limitado el poder sobre sus dominios».

iudgar, iulgar < JUDICARE, v. trans., «juzgar».

iuntancia < JUNTANTIA, sust, fem. sing., «unión».

iur, iuro < JURE, sust. masc. sing., «derecho, poder».

iuramentar < JURAMENTARE, v. intr., «jurar».

iuramiento, derivado de JURARE, sust. masc. sing., «juramento».

iurar < JURARE, v. trans., «jurar».

iusticia < JUSTITIA, sust. fem. sing. «justicia».

iusticiar < $<$ IUSTITIARE, v. trans., «justiciar». iuyçio < IUDICIU, sust. masc. sing., «pleyto».

iuyz. < JUDICE, sust. masc. sing., «juez».

ladron < LATRONE, sust. masc. sing., «ladrón».

[lagar $]$ < PLAGARE, v. trans., «llagar, herir».

lamar < CLAMARE, v. trans., "convocar»; cfr. xamar.

lazeria < "LACERIA, sust. fem. sing., «molestia, incumbencia».

lediar, lidiar < LITIGARE, v. intr., «pelear, contender».

lee, ley < LEGE, sust, fem. sing., «ley».

[legittimo] < LEGITIMU, adj. masc. sing. «legítimo».

lego, leygo < LAICU, sust. masc. sing., «laico».

$\mid$ leygal $\mid<$ LEGALE, adj. sing., «legal».

$\mid$ libra $\mid<$ LIBRA. sust. fem. sing., «libra».

licencia < LICENTIA, sust. fem. sing., «licencia, permiso».

lid, lide, lit < LITE, sust. fem. sing., «pelea, contienday.

linache. linage. del catalán arcáico llinyatge, sust masc. sing., «linaje».

livrar< LIBERARE, v. trans., «liberar».

madrastra, derivado de MATRE, sust. fem. sing, «madrastra».

$\mid$ maldecir $\mid<$ MALEDICERE, v. trans., «maledecir».

malfechor < *MALEFACTORE, sust. masc. sing., «malfechor».
$[$ malfetria $]<*$ MALEFACTORIA, sust. fem. sing., «delito, maldad».

malquerencia $<$ MALEQUAERENTIA. sust. tem. sing., «malevolencia, animosidad».

mancebo $<$ * MANCIPU, sust, masc. sing., «muchacho», documentado a menudo con el valor de «criado».

manda, derivado de MANDARE, sust. fem. sing., «documento, legado. cesión, donación, testamento».

mandadero < MANDATARIU, sust. masc. sing., «mensajero, alguacil, legado».

mandado, derivado de MANDARE, sust. masc. sing., «orden, disposición».

[mandador], derivado de MANDARE, sust. masc. sing., «mensajero, alguacil, legado».

mandamiento, derivado de MANDARE. sust. masc. sing., «orden, disposición».

mandar < MANDARE, v. Irans., «mandar, disponer».

$[$ manear $]<$ MANEARE, v. trans., «Usar, manejar».

[maordomo], mayordomo < $\mathrm{MAJOR}$ DOMU, sust. mase. sing., «mayordomo».

mayor < MAJORE, sust. mase. sing., «grande del reino, dignatario de corte»; adj. mase. sing., «mayor».

mayoral $<$ MAJORALE, sust. masc. sing., documentado con el valor de: «algualcil» y «grande del reino»; también con valor de adjetivo: «de ilustre linaje».

meaya < MEDALIA, sust. fem. sing., «medalla» (noneda antiguamente en uso que valía la sexta parte de un dine? o medio moravedi).

meder < METIRI, v. trans., «medir, estimar, valuars.

mengua, mingua $<$ "MINUA, sust. fem. sing., «reducción, disminución, escasez».

menor < MINORE, sust. masc. sing., «hombre perteneciente a una cepa social no elevada»; adj. masc. sing., «minor».

mentira, derivado de MENTIRE, sust. fem. sing., «mentira».

mentirero, derivado de MENTIRE, sust. masc. sing., «mentiroso». 
mercadero < MERCATARIU, sust. masc. sing., «mercante, comerciante».

mercador, del catalán antiguo mercader, sust. masc. sing., «mercante, comerciante».

merced, mercee, mercet $<$ MERCEDE, sust. fem. sing., «merced».

mercendero, derivado de MERCEDE, sust. masc. sing., «criado asalariado».

merchandia, francesismo derivado de MERCATU, sust. fem. sing., «mercancía».

merino < MAJORINU, sust. masc. sing., «gobernador».

mesquino, del árabo miskîn, sust. ed adj. masc. sing., «pobre, indigente».

meyorar < MELIORARE, v. trans., «mejorar»; cfr. mellorar.

meyoria, derivado de MELIORE, sust. fem. sing., «mejoramiento, incremento».

mezquindat, derivado del árabo miskîn, sust. fem. sing., "pobreza, indigencia».

[minguar], derivado de "MINUA, v. trans., «desminuir, reducir, escasear».

ministro, derivado de MINISTRARE, «ministro».

moravedi, del árabo murabitî, sust. masc. sing., «maravedi» (moneda antiguamente en uso, tuvo valores distintos, acuñada en cobre, plata, oro).

$\mid$ nacion $\mid<$ NATIONE, sust. fem. sing., «nación».

negar < NEGARE, v. trans., «negar».

negligencia $<$ NEGLIGENTIA, sust. fem. sing., «negligencia».

negocio < NEGOTIU, sust. masc. sing., «negocio, trato».

nemiga < INIMICA, sust. fem. sing., «agravio, tuerto, injusticia».

nomnar < NOMINARE, v. trans., «nombrar».

$\mid$ notario $\mid<$ NOTARIU, sust. masc. sing., «notario».

nozimiento $<{ }^{*}$ NOCEMENTU, sust. masc. sing., «daño, perjuicio».

obispado, derivado de EPISCOPU, sust. masc. sing., «obispado».

obligacion < OBLIGATIONE, sust. fem. sing., «obligación».

[obrero], derivado de OPERA, sust. masc. sing., «obrero». omazio, omecio, omezio, omezio < HOMICIDIU, sust. masc. sing., «homicidio». omenage, del occitánico omenatge, sust. masc. sing., «omenaje».

[omiciero], [omiçiero], [omiziero], derivado de HOMICIDIU, sust. masc. sing., «homicida».

onça< UNCIA, sust. fem. sing., «onza». orden < ORDINE, sust. fem. sing., «orden, mando, imposición».

ordenamiento, derivado de ORDINE, sust. masc. sing., «orden, mando, imposición». ordenar < ORDINARE, v. trans., «mandar, imponer».

osamiento, dervato di AUSARE, sust. masc. sing., «osadía».

osmar <*ADESTIMARE, v. trans., «valutar, estimar», cfr. asmar.

otorgamiento, derivado de *AUCTORICARE, sust. masc. sing., «concesión, permiso».

otorgar, outorgar < AUCTORICARE, $v$. trans., «conceder, permitir».

padron < PATRONU, sust. masc. sing., «dueñ, propietario».

pagar < PACARE, v. trans., «pagar».

paladinamientre, derivado de PALAM, adv. de modo, «publicamente, abiertamente».

paladino (en), derivado de PALAM, locución adverbial, «publicamente, abiertamentes.

parcionero < *PARTIONARIU, sust. masc. sing., «compañero, cómplice».

parcir < PARCERE, v. trans., «perdonar». partecion, particion $<$ PARTITIONE, sust. fem. sing., "partició, división».

partemiento < ${ }^{*}$ PARTIMENTU, sust. masc. sing., "partición, división».

partilla, partiya, partya $<$ PARTICULA, sust. fem. sing., «parte».

partir < PARTIRE, v. trans., «partir, dividir».

pasco, derivado de PASCERE, sust. masc. sing., «pasco».

patrimonio < PATRIMONIU, sust masc. sing., «patrimonio, bienes».

pechar < "PACTARE, v. trans., "pagar». pecunia, derivado de PECU, sust. fem. sing., «pecunia, dinero».

peguyar, peguiar < *PECULIARE, sust. masc. sing., «bienes». 
peguyo, peguyu < PECULIU, sust. masc. sing., «bienes».

pena < POENA, sust. fem. sing., «pena, condena».

penar, derivado de POENA, v. trans., «condenar».

penedencia < PENITENTIA, sust. fem. sing., «penitencia».

penedencial < PENITENTIALE, sust. fem. sing., «penitente».

penno < PIGNU, sust. masc. sing., «prenda».

pennora, derivado de PIGNEARI, sust. fem. sing., "prenda, garantía».

pennorar < PIGNERARI, v. trans., «embargar, secuestrar».

perdon, derivado de PERDONARE, sust. masc. sing., «perdón».

perdonar < PERDONARE, v. trans., "perdonar".

periurar.se $<$ PERJURARE, v. intr., «perjurar».

periurio, periuro, derivado de PERJURARE, sust. masc. sing., «perjuro».

personero < PERSONARIU, sust. masc. sing., «procurador».

[pertenecer $\mid<$ PERTINERE, v. trans.. «pertenecer».

[pertenencia], derivado de TENERE, sust. masc. sing., «posesiones».

pesquerir < *PERQUIRERE, v. trans., «verificar, indagar».

pesquisa, part. pas. sustant., ver pesquerir, «inspección, pesquisa».

plaço, plazo, prazo < PLACITU, sust. masc. sing., «plazo».

planar < PLANARE, v. trans., «aplanar, aclarar».

pletear, $\mid$ preytear $\mid<{ }^{*} \mathrm{PLACITEARE}, \mathrm{v}$. trans., «pletear, pactar».

[pleytés], derivado de PLACITU, sust. masc. sing., «cada una de las partes en el pleito».

pleyto < PLACITU. sust. masc. sing., «causa, juicio».

poderio, derivado de POTERE, sust. masc. sing., «poder, autoridad».

porfia < PERFIDIA, sust. fem. sing., documentado con el valor originario de «malvestad».

[porfiar|, derivado de PERFIDIA, v. intr., «insistir».

poridat $<$ PURITATE, sust. fem. sing., en
Ia locución adverbial en poridat significa «secretamente, íntimamente».

possession < POSSESSIONE, sust. fem. sign., «posesión».

postiya < POSTILLA, sust. fem. sing., «atribución».

[pozonador] < POTIONATORE, sust. masc. sing., «envenenador».

preciar, derivado de PRETIU, v. Irans., «valutar, estimar».

premia, derivado de PREMERE, sust. fem. sing., «costricción, presión».

presentar.se < PRESENTARE, v. trans. y pronom., «presentarse».

presion < PREHENSIONE, sust. fem. sing., "cárcel».

preso, derivado de PREHENDERE, sust. masc, sing., «preso».

prestamo, derivado de PRAESTARE, sust. masc. sing, "préstamo».

prestar < PRAESTARE, v. trans., «prestar».

preteamiento, derivado de PLACITU, sust. masc. sing., «compromiso, acuerdo».

privilegio < PRIUILEGIU, sust. masc. sing., «privilegio».

procurador < * $\mathrm{PROCURATORE,} \mathrm{sust.}$ masc. sing., «procurador».

prod, proe, prol $<$ PRODE, sust. fem. sing.. «provecho, ventaja».

prolongar, prelongar, pelongar $<\mathrm{PRO}-$ LUNGARE, v. trans., "detardar, posticipar».

prolongançia, derivado de PROLUNGARE, sust. fem. sing., «retraso».

prometemiento, derivado de PROMI-

TTERE, sust. masc. sing., «promesa». promission, derivado de PROMITTERE, sust. fem. sing., «promesa».

propinco, $[$ propincu $]<$ PROPINQUU, sust. y adj. masc. sing., «parente próximo, próximo».

proponemiento, derivado de PROPONERE, sust. masc, sing., «propósito, intención».

prova, derivado de PROUARE, sust. fem. sing., «prueba».

provar $<$ PROUARE, v. trans., «probar, demostrar».

[provecho] < PROFECTU, adj. masc. sing., «provechoso, ventajoso».

[provechoso] < PROFECTOSU, adj. masc. sing., "provechoso, ventajoso». 
provision < PROUISIONE, sust. fem. sing., «acción y resultado de proveer».

purgar.se < PURGARE, v. trans., «justilicarse, demostrarse inocente».

[quebrantamiento], derivado de CREPARE, sust. masc. sing., «quebranlamiento, quiebra, daño».

[quebrantancia], derivado de CREPARE, sust. lem. sing. «quebrantamiento, quiebra, daño».

quebrantar, derivado de CREPARE, $v$. trans., «contravenir».

[quemador], derivado de CAIMARE, sust. masc. sing., «incendiario».

quemar<* CAIMARE, v. trans., «quemar, incendiar».

querella $<$ QUERELLA, sust. fem. sing., «Causa, disputa, contención, contienda».

querellador, derivado de QUERELLA, sust. masc. sing., "el que se querella, que pide justicia».

querellar.se, derivado de QUERELLA, $v$. pronom., «quejarse, pedir justicia».

querelloso, derivado de QUERELLA, sust. masc. sing., uel que se querella, que pide justicia».

quexar.se < QUASSIARE, v. pronom., «quejarse».

quinon < QUINIONE, sust. masc. sing., «parte que le corresponde a cada uno «le algo poseído entre varios».

quitamiento, derivado de QUIETU, sust. masc. sing., «perdón de una deuda o de parte de ella que hace el acreedor». quito, derivado de quitar, adj. masc. sing., «libre, disculpado».

recabdo, recaudo < RECAPITU, sust. masc. sing., «recaudo, garantía».

recebimiento, derivado de RECIPERE, sust. masc. sing., «rapto».

recodir < *RECUTERE, v. intr., «llegar a un sitio, alcanzar».

recombrar < RECUPERARE, v. trans., «recobras».

refulgancia, deivalo di REFUSARE, sust. fem. sing., «rechazo».

refrenar, derivado de FRENU, v. trans., «refrenar».

refugar $<$ REFUSARE, v. trans., «rechazar»》. responder < RESPONDERE, v. intr., «contestar».

retraer, derivado de TRAHERE, $\vee$ trans., «quitar, abonar».

reverencia < REUERENTIA, sUst. fem. sing., «reverencia».

[revolver], derivado de UOLUERE, $v$. trans., «volver a discutir».

[riegla $]<$ REGULA, sust. fem. sing., «regla, disposición».

roba, derivado del germánico raubôn, sust. fem. sing., «cosa robada».

robador, derivado del germánico raubôn, sust. masc. sing., "ladrón».

robar, derivado del germánico raubôn, $v$. trans., «robar».

|rovlar $|$,$| rovrar \mid<$ ROBORARE, v, trans. «firmar, confirmar un documento».

sabidor, derivado de SAPERE, sust mase. sing., «quien esté enterado de cierta cosa».

[sagrar , derivado de SACRU, v. trans., «consagrar».

sayon, del gótico *sagjis, sust. masc. sing., «empleado de justicial encargado de hacer las citaciones y ejecutar los embargos».

scripto < SCRIPTU, sust. masc. sing., «documento, acta notarial».

scriptura, derivado de SCRIBERE, sust. fem. sing., «documento, acta notarial».

[seellar $]<*$ SIGILLARE, v. trans., «sellar».

seello $<$ SIGILLU, sust. masc. sing., «se110 ».

seglar, derivado de SAECULU, adj. masc. sing., «laico».

[senescal], del germánico siniska!k, sust. masc. sing. «jefe de la nobleza, que la gobernaba especialmente en la guerra».

sennero, sinero < SINGULARIU, adj. masc, sing., «solo, individualmente».

sennorio, derivado de SENIORE, sust. masc. sing., «poder»; documentado con el valor de «prestar servicio» en la expresión aver sennorio.

sentencia < SENTENTIA, sust. fem. sing., «sentencia».

[sentenciar], derivado de SENTENTIA, v. trans., «juzgar».

servedumne $<$ SERUITUMINE, sust. fem. sing. «servidumbre, esclavitud». 
servicio < SERUITIU, sust. masc. sing., «servicio».

[silogismu] < SYLLOGISMU, sust. masc. sing, «silogismo».

sinnal, derivado de SIGNU, sust, mase. sing., «señal».

sinnalar, derivado de SIGNU, v. trans., «señalar, marcar».

sobreuiant, derivado de *SUPERPODIARE, adj. masc. sing., «poderoso, excelenter.

[sodomitico|, derivado de SODOMITA, sust. masc. sing., «sodomita».

soescrevir, derivado de SCRIBERE, $v$. trans.. «subscribir».

sofismo $<$ SOPHISMA, sust. masc. sing., «sofisma».

soldada, derivado de SOLDU, sust. fem. sing., «sueldo».

soltamiento, derivado de SOLUERE, sust. masc. sing.. «acción y resultado de liberar».

soltar, derivado de SOLUERE, $v$. trans., «desatar, liberar».

solver < SOLUERE, v. trans., «relevar, soltar».

someter < :SUBMITTERE, v. trans., «someter».

sospecha < SUSPECTA, sust. fem. sing., «sospecha».

sospechoso, derivado de SUSPECTARE, adj. masc. sing., «sospechoso».

sustimiento < "SUSTIMENTU, sust. masc. sing. «sustento, mantenimiento».

\{subiecto\}, derivado de SUBJACERE, part. pas. sustant., «súbdito».

subtiliçar, derivado de SUBTILE, v. intr., «sutilizar, cavilar».

sucidumne $<$ "SUCITUMINE, sust. fem. sing., «acción deshonesta».

summa < SUMMA, sust. fem. sing., documentado con el valor de «suma, cantidad de dinero» y de «suma, operación aritmética».

tardar < TARDARE, v, intr., «detardar». [templar $\},\{$ temprar $\mid<$ TEMPERARE, $v$. trans., «moderar, mitigar»,

[temporal], derivado de TEMPL, adj. masc. sing. «temporal».

tempramiento < TEMPERAMENTU, sust. masc. sing., «moderación, templanza». tenedor, derivado de TENERE, sust. masc. sing., «depositario».

tenemiento, derivado de TENERE, sust. masc. sing., «custodia».

tenplancia < TEMPERANTIA, sust. fem. sing., «moderación, templanza».

termino < TERMINU, sust. mase. sing., «plazo, término».

testamento $<$ TESTAMENTU, sust. masc. sing., «testamento».

testigo, derivado de TESTIFICARE, sust. masc. sing., «testigo».

testimoniar <*TESTIMONIARE, v, intr., «testimoniar».

testimonio, testimonia < TESTIMONIU, sust. masc. sing., «testigo».

tirar $<$ TIRARE, v. trans., «quitar, llevarse».

toller, toyer < TOLLERE, v. trans., «quitar, llevarse».

tormentamineto, derivado de TORMENTARE, sust. masc. sing., «tormento». tormentar < TORMENTARE, v. trans., «tormentar».

tormiento < TORMENTU, sust. masc. sing., «tormento».

tornar < TORNARE, v. intr., «devolver».

[torticero], [tortizero] < *TORTICEARIU, adj. masc. sing., «injusto, errado».

tractado < TRACTATU, sust. masc. sing., «tratado».

tractar < TRACTARE, v. trans., «tratar». traslado $<$ TRANSLATU, sust. masc. sing., «ejemplar de un documento».

[trasmudar], derivado de MUTARE, v. trasn., «cambiar».

[traspassar], derivado de PASSARE, v. trans., «contravenir».

trastornar, derivado de TORNARE, $v$. trans., «molestar, destorbar».

traycion < TRADITIONE, sust. fem. sing., «traición».

tregua, dal gotico triggwa, sust. fem. sing., «tregua».

tributo < TRIBUTU, sust. masc. sing., «tributo, impuesto».

tuerto < TORTU, sust. masc. sing., «tuerto».

usar< USARE, v. trans., «usar, emplear». usura < USURA, sust. fem. sing., «usura». usurero, derivado de USURA, sust masc. sing., «usurero».

uueste < HOSTE, sust. fem. sing., «ejército, milicia»; cfr. hoste, hueste. 
[valadero|, derivado de UALERE, adj. masc. sing., «valable».

valer < UALERE, v. intr., «valer».

vassallo, del céltico *yassallos, sust. masc. sing., «vassallo».

vecino < UICINU, sust. mase. sing., «vecino».

[vedar] < UETARE, v. trans., «vedar».

vencedor, derivado de UINCERE, sust. masc. sing., «vencedor».

vencimiento, derivado de UINCERE, sust. masc. sing., «victoria».

vendecion, derivado de UENDERE, sust. fem. sing., «venta».

vendedor, derivado de UENDERE, sust. masc. sing., «vendedor».

vender < UENDERE, v. trans., «vender». venzer < UINCERE, v. trans., «vencer».

verdadero, derivado de UERITATE, adj. masc. sing., "verdadero». vigilar < UIGILARE, v. trans., «vigilar, controlar».

$v i l<$ UILE, adj. masc. sing., «vill».

[villano] < UILLANU, sust. masc. sing., «villano, hombre del pueblo».

vozero, derivado de UOCE, sust. masc. sing., "portavoz, quien habla en nombre de otro u otros»; adj. masc. sing., «incoherente».

$x a m a r<$ CLAMARE, v. trans., «convocar»; cfr. lamar.

yerro, derivado de ERRARE, sust. masc. sing., «yerro».

ygual < AEQUALE, adj. mase. sing., «igual».

ygualdade, derivado de AEQUALE, sust. fern. sing., «igualdad». 